\title{
NRPI is a Prognostic Factor and Promotes the Growth and Migration of Cells in Intrahepatic Cholangiocarcinoma
}

This article was published in the following Dove Press journal: Cancer Management and Research

\author{
Yong-Na Wu iD ${ }^{1-4, *}$ \\ Li-Hong $\mathrm{He}$ iD $1,2,5, *$ \\ Zhong-Tian Bai (iD) ${ }^{1,2,5, *}$ \\ Xun Li iD 1,2,5
}

'Department of General Surgery, The First Hospital of Lanzhou University, Lanzhou 730000, Gansu Province, People's Republic of China; ${ }^{2}$ Key Laboratory of Biological Therapy and Regenerative Medicine Transformation Gansu Province, Lanzhou 730000, Gansu Province, People's Republic of China; ${ }^{3}$ Northwest Institute of Eco-Environment and Resources, Chinese Academy of Sciences, Lanzhou 730000, Gansu Province, People's Republic of China; ${ }^{4}$ University of Chinese Academy of Sciences, Beijing 100049, People's Republic of China; ${ }^{5}$ The First Clinical Medical College, Lanzhou University, Lanzhou 730000, Gansu Province, People's Republic of China

*These authors contributed equally to this work
Correspondence: Xun Li

Department of General Surgery, The First Hospital of Lanzhou University, Lanzhou University, No. I Donggang West Road, Lanzhou 730000, Gansu Province,

People's Republic of China

Email Ixdr2I@I26.com
Background: Neuropilin-1 (NRP-1) participates in cancer cell proliferation and metastasis as a multifunctional co-receptor by interacting with multiple signaling pathways. However, few studies have addressed the precise function and prognosis analysis of NRP1 in intrahepatic cholangiocarcinoma (ICC). We aimed to study the correlations between NRP1 and clinicopathological characteristics and NRP1 effect on ICC cell line functions.

Methods: NRP1 mRNA and its protein levels in human ICC tissues and cell lines were detected by IHC, qRT-PCR, and WB method. Transwell, wound healing, and CCK-8 assays were performed to verify the effects of NRP1 knockdown and overexpression on cell migration and proliferation capability.

Results: NRP1 proteins and mRNA levels increased in ICC tissues compared to those in paired adjacent non-tumor tissues. High NRP1 expression of ICC tissues was related to poor prognosis. NRP1 expression level was expected to be an independent prognosticator for overall survival and cumulative tumor recurrence, and was closely related to tumor number $(P=0.047)$. Knockdown of NRP1 inhibited cell proliferation and migration capability of RBE cells in vitro, and NRP1 overexpression in 9810 cells accelerated proliferation and migration. Additionally, NRP1 may promote cell proliferation and migration in ICC via the FAK/PI3-K/ AKT pathway.

Conclusion: As an oncogene, NRP1 may function as a candidate target and prognostic biomarker of value for ICC therapy.

Keywords: neuropilin-1, prognostic factor, cell growth and migration, intrahepatic cholangiocarcinoma

\section{Introduction}

Intrahepatic cholangiocarcinoma (ICC), known as the second most common liver cancer, accounts for approximately $10 \%$ of primary liver cancer. Most ICCs originate from intrahepatic bile duct epithelial cells, with strong metastatic and invasion ability and poor prognosis. ${ }^{1,3}$ Currently, the best way to treat ICC is through surgical resection; however, only $15 \%$ of ICC patients present with resectable ICC. ${ }^{4,5}$ In addition, radiotherapy and chemotherapy offer limited benefits for advanced ICC. The mortality and incidence of ICC is on the rise worldwide, and the median survival is within 3 years, seriously endangering human health. ${ }^{6}$ Therefore, identification of novel therapeutic molecular targets is essential for improving the prognosis of ICC. ${ }^{7,8}$

NRP1 was first discovered as an adhesion molecule in the frog nervous system in $1987 .{ }^{9}$ It is a non-tyrosine kinase transmembrane glycoprotein located on the cell 
surface and plays a role of a co-receptor for secreted Semaphorin-3A (Sema3A). Previous studies have shown that NRP1 gene expression is widespread over a variety of cells and tissues such as endothelial cells, and the heart, liver, lung, kidney, pancreas, and skeletal muscle. ${ }^{10}$ NRP1 plays a vital role in the nervous, digestive, and immune systems, etc. through interaction with various ligands, promoting angiogenesis, neural development, cytoskeleton remodeling, inflammation, initial immune response, as well as occurrence and development of tumors. ${ }^{11,14}$ Noteworthily, the role of NRP1 in the occurrence and development of human malignant tumors has arisen as an interesting research topic recently. ${ }^{15,16}$ Increasing evidence suggests that higher expression or mutations of NRP1 are closely related to initiation, progression, and prognosis of varieties of types of malignant tumors in humans, including hepatocellular carcinoma, ${ }^{17}$ gastric cancer, ${ }^{18}$ breast cancer, ${ }^{19,20}$ prostatic cancer, and pancreatic cancer. ${ }^{21,22}$

However, so far the expression of NRP1 and its underlying role in the occurrence, development, and prognosis of ICC have been rarely discussed to date. The important role of NRP1 in many malignant tumors has prompted us to investigate the biological effects and clinical relevance of NRP1 in human ICC. In comparison with paired adjacent non-tumor tissues, we found significantly increased expression of NRP1 protein in the tumor tissues of ICC patients. We also found that the upregulation of NRP1 was an independent prognosticator for overall survival and cumulative recurrence. In addition, in vitro experiment indicated that NRP1 depletion significantly inhibited the proliferation and migration capability of human ICC cell lines; however, overexpression of NRP1 resulted in opposite results. Based on these results, we conducted further experiments and found that NRP1 possibly enrolled in the net of the FAK/PI3-K/AKT pathway. To sum up, NRP1 is a valuable promoting factor in ICC and a potential target for further drug development.

\section{Materials and Methods}

\section{Patients and Clinical Tissue Samples}

Tumor tissues and paired adjacent non-tumor tissues were collected from 291 ICC patients treated at Zhongshan Hospital of Fudan University from 2010 to 2014. All patients' tissue specimens were classified as ICC through pathological examination. Fresh tissue specimens were obtained, formalin-fixed, and paraffin embedded according to a previously reported method. ${ }^{23}$ The study was approved by the Ethics Committee, and all the participants provided informed consent under Institutional Review Board protocols before tissue specimen collection. Patients' clinicopathological data including gender, age, tumor number, tumor size, embolus, tumor encapsulation, TNM, liver cirrhosis, NRP1, lymphonodus, capsule, serum alpha fetoprotein (AFP), CA199, and HBsAg concentration were collected before surgery for analysis.

\section{Immunohistochemistry (IHC)}

IHC was conducted on the basis of a formerly reported method with slight modifications. ${ }^{24,25}$ In brief, tissue microarrays were constructed using BioChip (Shanghai, China). The rabbit monoclonal antibody against human NRP1 (1:200 dilution) was purchased from Abcam (Cambridge, UK). The treated sections were placed under an optical microscope (Olympus Corp., Tokyo, Japan; magnification, $\times 200$ ) to observe the experimental results. For comprehensive analysis of the results, in terms of staining intensity and stained cell quantity, two specialist pathologists who lacked information about the patients separately evaluated the tissue sections. The intensity was scored as follows: 0 , negative; 1 , weak; 2 , moderate; and 3 , strong. The frequency of positive cells was defined as follows: 0 , less than $5 \% ; 1,5-25 \% ; 2,26-50 \% ; 3,51-$ $75 \%$; and 4 , greater than $75 \%$.

\section{ICC Cell Lines and Culture}

Human RBE and 9810 ICC cell lines were obtained from Shanghai Cell Bank (Shanghai, China). They were cultured under standard condition using Dulbecco's modified Eagle's medium (DMEM; Gibco, Shanghai, China) complemented with $10 \%$ fetal bovine serum (FBS; Gibco) and $1 \%$ Streptomycin-Penicillin (100 IU/mL; Gibco) at $37^{\circ} \mathrm{C}$ with $5 \% \mathrm{CO}_{2}$. This was followed by 1-day invasion and migration assays, 2-day qRT-PCR and WB analysis, 3-day lentivirus transfection and CCK-8 assay experiments. All operations were conducted in a biosafety cabinet after ultraviolet sterilization.

\section{Cell Transfection}

The lentiviral packaging system was composed of the vectors pGCL-puro, pHelper 1.0, and pHelper 2.0, provided by Hanyin Biotech Genechem (Shanghai, China). Three plasmids (pHelper 1.0, pHelper 2.0, and pGCL-puro or pGCL-puro-NRP1) were co-transfected into $293 \mathrm{~T}$ cells using Lipofectamine 2000 (Invitrogen, USA). After 12 hours, the cell culture medium was replaced with fresh 
medium containing $10 \%$ fetal calf serum. The lentivirus supernatant containing NRP1 cDNA was collected at 48 hours after co-transfection. Lentivirus supernatant was transfected into 9810 cells with $8 \mu \mathrm{g} / \mathrm{mL}$ polybrene (Sigma-Aldrich, ST. Louis, MO, USA) to obtain a stable cell line, named 9810-OE, the $9810-\mathrm{NC}$ was used as a control. A set of shRNA (short hairpin RNA) lentiviral vectors differed in NRP1-targeting sequences (Supplementary Table S1) and pLKO.1-puro empty vector with green fluorescent protein (GFP) were purchased from Genechem (Shanghai, China). The lentiviral packaging system was the same as mentioned above. Lentivirus supernatant was transfected into RBE cells with $8 \mu \mathrm{g} / \mathrm{mL}$ polybrene (Sigma-Aldrich) to obtain a stable cell line, named RBE-shNRP1. The RBE-NC was used as a control.

\section{Quantitative Real-Time Polymerase Chain Reaction (qRT-PCR)}

In our study, qRT-PCR was performed according to a previously reported method. ${ }^{22}$ NRP1 and GAPDH (inner control) primers were synthesized and purchased from Sangon Biotech (Shanghai, China): NRP1 sense, 5'ATCACGTGCAGCTCAAGTGG-3', and antisense, 5'-TC ATGCAGTGGGCAGAGTTC-3'; GAPDH sense, 5'-GG TATGACAACGAATTTGGC-3', and antisense, 5'GAGCACAGGGTACTTTATTG-3'. The experiments were repeated in triplicate.

\section{WB Analysis}

In this study, WB was performed according to a previously reported method with slight modification. ${ }^{24,25}$ Briefly, RIPA lysis buffer containing protease and phosphatase inhibitors for extracting total protein from tissues and cells was purchased from SigmaAldrich. Anti-human NRP1 antibody (1:2000 dilution) was purchased from Abcam. Finally, relative proteins were quantified using Image-pro plus 6.0 (Media Cybernetics, USA).

\section{Cell Proliferation, Invasion, and Migration Assays}

To explore cell proliferation activity, we performed CCK8 , assay experiments in six replicates. To evaluate cell migration capability, we performed transwell and wound healing assays separately, and the experiments were performed in triplicate. CCK-8, transwell, and wound healing assays were carried out according to a previously reported method. ${ }^{24}$ We blocked proliferation in the wound healing assays with $2 \%$ serum concentration. The variation in migration capability of cells can be determined by observing and photographing using a light microscope (Olympus Corp.; magnification, $\times 100$ ).

\section{Statistical Analysis}

Data were analyzed using SPSS v.21.0 software (IBM Corp., Chicago, IL, USA). The correlation between NRP1 expression and clinicopathological characteristics were analyzed using Fisher's exact test or Pearson chisquared test. We used multi-factor or one-way ANOVA to analyze the significance of differences among groups. Overall survival and cumulative recurrence were described using Kaplan-Meier plots (Log rank tests). The data for cell functional assays, described as means \pm standard deviation, were evaluated by Mann-Whitney $U$-test. $P<0.05$ was regarded as representing a statistically significant difference.

\section{Results \\ Expression of NRPI in ICC Tissues and Its Correlation with Prognosis and Clinicopathological Characteristics}

Figure 1 shows the immunohistochemical expression of NRP1 in ICC tissues and adjacent non-tumor tissues. Light microscopy showed that the expression levels of NRP1 protein between ICC and adjacent tissues were different. In total, 188 samples $(64.60 \%)$ showed high NRP1 expression, while 103 samples (35.40\%) had low NRP1 expression. The overall survival and cumulative tumor recurrence in ICC patients were evaluated using KaplanMeier analysis, thus revealing that high NRP1 expression was significantly linked with decreased overall survival and high risk of cumulative tumor recurrence (Figure 2). Further, the high NRP1 expression always indicates a poor prognosis. The results of correlation between NRP1 expression and clinicopathological characteristics (Table 1) showed that NRP1 upregulation was significantly associated with tumor number $(P=0.047)$. No significant difference showed in age, gender, tumor size, AFP expression, CA199 expression, and TNM of NRP1 expression in patients with ICC $(P>0.05)$. Furthermore, univariate and multivariate Cox regression analyses (Table 2) demonstrated that NRP1 is an independent prognosticator of overall survival $(P=0.000)$ and cumulative tumor recurrence $(P=0.021)$. 
A

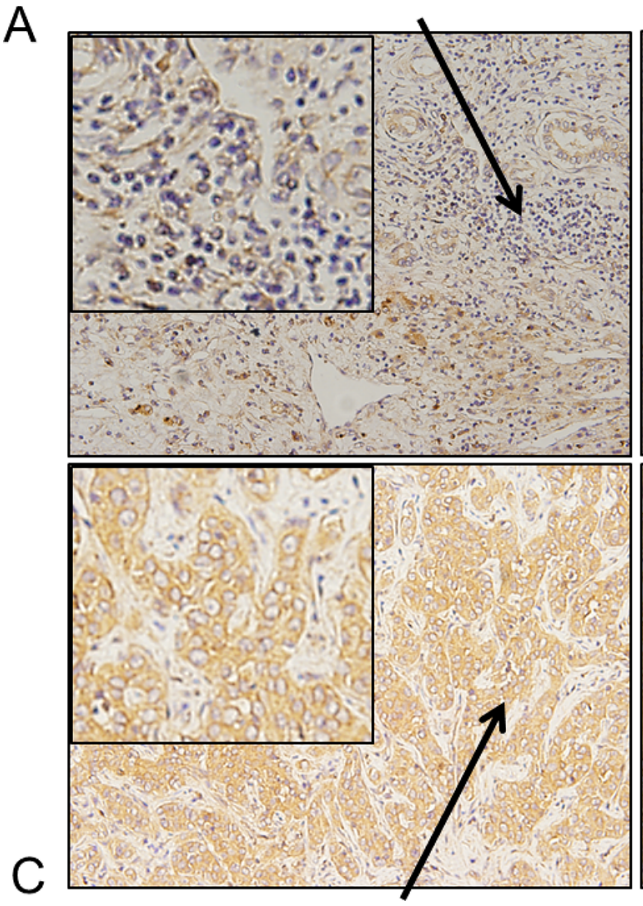

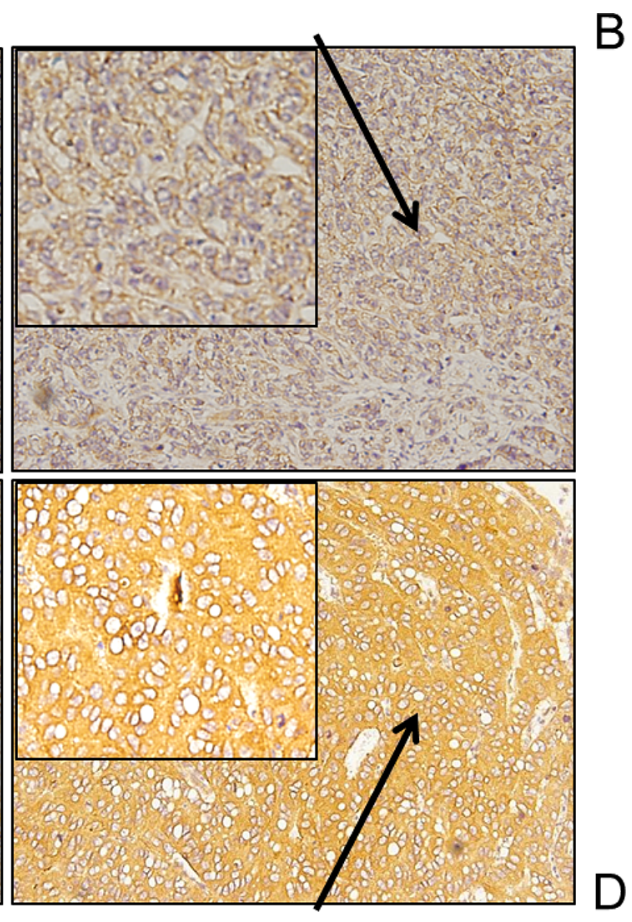

Figure I NRPI expression detection in both ICC tissues and adjacent non-tumor tissues using IHC analysis; Magnification, $\times 200$. (A) The staining in adjacent non-tumor tissue is weak, indicating that the expression level of NRPI is low. (B) Weak staining in tumor tissues, indicating that the expression level of NRPI is low. (C) Moderate staining in tumor tissues, indicating the general expression level of NRPI. (D) Strong staining in tumor tissues, indicating that the expression level of NRPI is high.
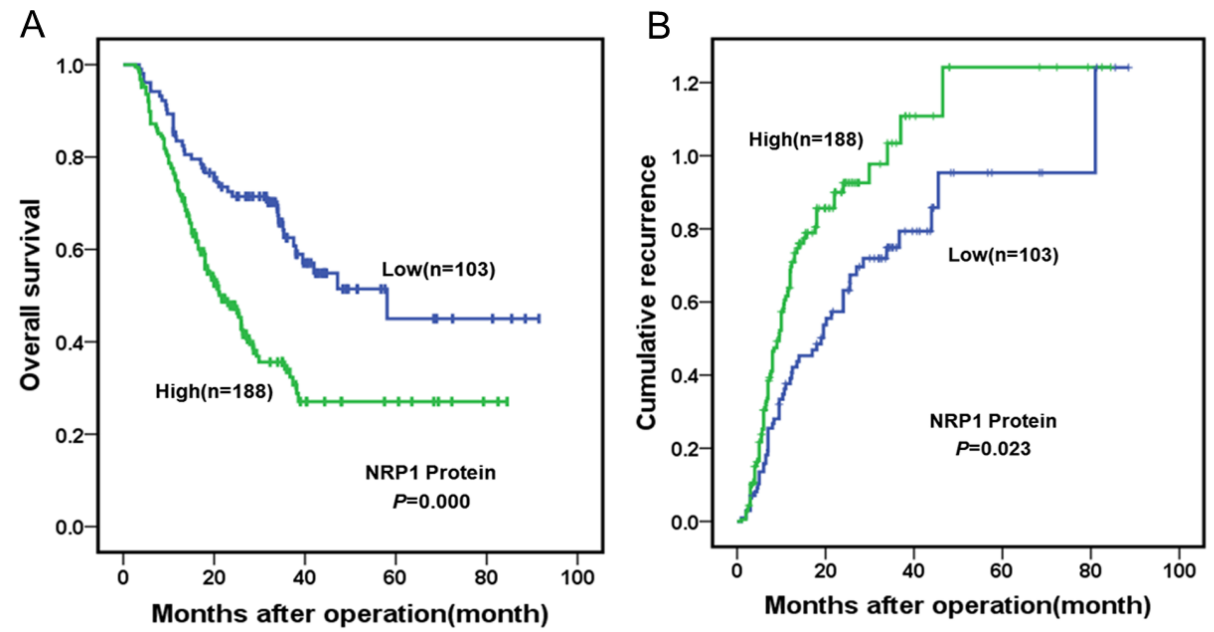

Figure 2 IHC staining level showing overall survival and cumulative tumor recurrence in ICC patients, evaluated using Kaplan-Meier analysis. (A) The overall survival of I03 ICC patients (35.40\%) with low NRPI expression was higher than that of I88 ICC patients $(64.60 \%)$ with high NRPI expression (P<0.00I). (B) The cumulative recurrence of I03 ICC patients (35.40\%) with low NRPI expression was lower than that of I88 ICC patients (64.60\%) with high NRPI expression $(P=0.023)$.

\section{Expression of NRPI is Upregulated in Human ICCs}

The expression of NRP1 was markedly increased in ICC tissues than that in adjacent non-tumor tissues at protein and mRNA levels. Specifically, NRP1 mRNA expression in 55 ICC tissues and their paired adjacent tissues were evaluated by qRT-PCR. Among the 55 groups, cancer tissues had higher levels of NRP1 mRNA than those in adjacent tissues in 33 pairs $(60.00 \%)$ (Figure $3 \mathrm{~A}$ ). At the protein level, cancer tissues had higher expression levels of NRP1 proteins than those in adjacent tissues in eight out of 15 pairs (53.33\%) (Figure 3D and E). Also, we assessed NRP1 protein expression levels in ICC cell lines by Western blot (WB), which revealed that the highest 
Table I Correlations Between the Factors and Clinicopathological Characteristics in ICC $(n=29$ I)

\begin{tabular}{|c|c|c|c|c|}
\hline \multicolumn{2}{|c|}{ Clinicopathological Indexes } & \multicolumn{3}{|c|}{ NRPI } \\
\hline & & \multirow{2}{*}{$\begin{array}{l}\text { Low } \\
56 \\
47\end{array}$} & \multirow{2}{*}{$\begin{array}{l}\text { High } \\
96 \\
92\end{array}$} & \multirow{2}{*}{$\begin{array}{l}P \\
0.625\end{array}$} \\
\hline Age (years) & $\begin{array}{l}\leq 50 \\
>50\end{array}$ & & & \\
\hline Sex & $\begin{array}{l}\text { Female } \\
\text { Male }\end{array}$ & $\begin{array}{l}44 \\
59\end{array}$ & $\begin{array}{l}73 \\
115\end{array}$ & 0.534 \\
\hline $\operatorname{AFP}(\mathrm{ng} / \mathrm{mL})$ & $\begin{array}{l}\leq 20 \\
>20\end{array}$ & $\begin{array}{l}89 \\
11\end{array}$ & $\begin{array}{l}170 \\
16\end{array}$ & 0.529 \\
\hline CAI99 (ng/mL) & $\begin{array}{l}\leq 30 \\
>30\end{array}$ & $\begin{array}{l}46 \\
48\end{array}$ & $\begin{array}{l}94 \\
85\end{array}$ & 0.611 \\
\hline Tumor size $(\mathrm{cm})$ & $\begin{array}{l}\leq 5 \\
>5\end{array}$ & $\begin{array}{l}41 \\
62\end{array}$ & $\begin{array}{l}90 \\
98\end{array}$ & 0.218 \\
\hline Tumor number & $\begin{array}{l}\text { Single } \\
\text { Multiple }\end{array}$ & $\begin{array}{l}83 \\
20\end{array}$ & $\begin{array}{l}136 \\
52\end{array}$ & 0.047 \\
\hline Embolus & $\begin{array}{l}\text { No } \\
\text { Yes }\end{array}$ & $\begin{array}{l}89 \\
14\end{array}$ & $\begin{array}{l}159 \\
29\end{array}$ & 0.732 \\
\hline Capsule & $\begin{array}{l}\text { No } \\
\text { Yes }\end{array}$ & $\begin{array}{l}11 \\
92\end{array}$ & $\begin{array}{l}25 \\
163\end{array}$ & 0.580 \\
\hline TNM & $\begin{array}{l}\mathrm{I} / \mathrm{II} \\
\mathrm{III} / \mathrm{IV}\end{array}$ & $\begin{array}{l}84 \\
19\end{array}$ & $\begin{array}{l}140 \\
48\end{array}$ & 0.192 \\
\hline Lymphonodus & $\begin{array}{l}\text { No } \\
\text { Yes }\end{array}$ & $\begin{array}{l}88 \\
15\end{array}$ & $\begin{array}{l}152 \\
36\end{array}$ & 0.335 \\
\hline Liver cirrhosis & $\begin{array}{l}\text { No } \\
\text { Yes }\end{array}$ & $\begin{array}{l}79 \\
24\end{array}$ & $\begin{array}{l}133 \\
55\end{array}$ & 0.420 \\
\hline
\end{tabular}

NRP1 expression level was found in the RBE cell line, whereas the lowest was observed in the 9810 cell line (Figure 3B and C).

\section{NRPI Depletion Impairs Proliferation, Invasion, and Migration of RBE Cell Line in vitro}

To explore the role of NRP1 in ICC cell lines based on the above experimental results, we knocked out NRP1 from the RBE cell line having high expression of NRP1. As shown in Figure 4, the normal control group was defined as the RBE-NC group and the NRP1 depletion group was defined as the RBE-shNRP1 group. After silencing, the expression of NRP1 mRNA level in the RBE-shNRP1 group decreased significantly (Figure 4A). Figure 4C presents the validation of transfection efficiency at the protein level. Figure 4B shows CCK-8, Figure4D and E show transwell, and Figure4F and $G$ show wound healing assay results, respectively. CCK-8 assay was used to analyze cell proliferation. Transwell and wound healing assays were used to evaluate cell invasion and migration capability. The results revealed that eliminating NRP1 impairs proliferation, invasion, and migration in $\mathrm{RBE}$ cells.

\section{NRPI Overexpression Promotes Proliferation, Invasion, and Migration of 9810 Cell Line in vitro}

To further prove the role of NRP1 in ICC cell lines, we overexpressed NRP1 in the 9810 cell line, which has the lowest expression of NRP1. In Figure 5, the normal control group was defined as the $9810-\mathrm{NC}$ group and the NRP1 overexpression group was defined as the 9810OE-NRP1 group. After overexpression, the expression of NRP1 mRNA in the 9810-OE-NRP1 group increased significantly (Figure 5A). Figure 5C shows the validation of 
Table 2 Univariate and Multivariate Analyses of Prognostic Factors in ICC $(n=291)$

\begin{tabular}{|c|c|c|c|c|}
\hline \multirow[t]{2}{*}{ Variable } & \multicolumn{2}{|c|}{ Cumulative Recurrence } & \multicolumn{2}{|l|}{ Overall Survival } \\
\hline & HR (95\% Cl) & $\boldsymbol{P}$ & HR (95\% Cl) & $P$ \\
\hline \multicolumn{5}{|l|}{ Univariate analysis } \\
\hline Age, years $(\leq 50$ vs $>50)$ & $0.872(0.639-1.189)$ & 0.387 & $1.208(0.877-1.662)$ & 0.247 \\
\hline Sex (female vs male) & 1.051 (0.768-I.440) & 0.756 & $1.204(0.864-1.677)$ & 0.273 \\
\hline AFP, ng/mL ( $\leq 20$ vs $>20)$ & $0.948(0.548-1.64 I)$ & 0.850 & $0.966(0.557-1.676)$ & 0.902 \\
\hline Tumor size, $\mathrm{cm}(\leq 5$ vs $>5)$ & $1.334(0.977-1.822)$ & 0.070 & $1.507(1.086-2.091)$ & 0.014 \\
\hline Tumor number (single vs multiple) & $1.890(1.353-2.64 I)$ & 0.000 & $1.766(1.250-2.495)$ & 0.001 \\
\hline Embolus (no vs yes) & $1.586(1.072-2.346)$ & 0.021 & $1.202(0.776-\mid .86 I)$ & 0.409 \\
\hline Tumor encapsulation (complete vs none) & $1.505(0.898-2.524)$ & 0.121 & $1.357(0.796-2.313)$ & 0.262 \\
\hline TNM (I/II vs III/IV) & $2.009(1.424-2.834)$ & 0.000 & $2.480(1.764-3.486)$ & 0.000 \\
\hline NRPI (low vs high) & $0.688(0.496-0.955)$ & 0.025 & $0.452(0.314-0.652)$ & 0.000 \\
\hline Liver cirrhosis (no vs yes) & $1.256(0.896-1.760)$ & 0.186 & I.084 (0.757-I.552) & 0.661 \\
\hline Lymphonodus (no vs yes) & $2.529(1.749-3.657)$ & 0.000 & $2.946(2.046-4.242)$ & 0.000 \\
\hline CAI99, ng/mL ( $\leq 30$ vs $>30)$ & $1.280(0.934-1.754)$ & 0.125 & $1.586(1.131-2.222)$ & 0.007 \\
\hline HBsAg (no vs yes) & $0.895(0.652-1.228)$ & 0.491 & $0.712(0.509-0.996)$ & 0.047 \\
\hline \multicolumn{5}{|l|}{ Multivariate analysis } \\
\hline Tumor size, $\mathrm{cm}(\leq 5$ vs $>5)$ & & & $1.564(1.084-2.255)$ & 0.017 \\
\hline Tumor number (single vs multiple) & I.76I (I.25I-2.479) & 0.001 & $1.783(1.224-2.597)$ & 0.003 \\
\hline TNM (I/II vs III/IV) & $1.078(0.507-2.291)$ & 0.845 & $1.133(0.565-2.273)$ & 0.725 \\
\hline Lymphonodus (no vs yes) & $2.024(0.921-4.448)$ & 0.079 & $2.298(1.120-4.716)$ & 0.023 \\
\hline CAI99, ng/mL ( $\leq 30$ vs $>30)$ & & & $1.483(1.052-2.091)$ & 0.024 \\
\hline HBsAg (no vs yes) & & & $0.708(0.493-1.016)$ & 0.061 \\
\hline NRPI (low vs high) & $0.675(0.483-0.942)$ & 0.021 & $0.46 \mathrm{I}(0.3 \mathrm{I} 2-0.683)$ & 0.000 \\
\hline Embolus (no vs yes) & $1.429(0.955-2.138)$ & 0.083 & & \\
\hline
\end{tabular}

transfection efficiency at the protein level. Figure 5B shows the results of CCK-8, Figure 5D and E showthe results of transwell, and Figure $5 \mathrm{~F}$ and $\mathrm{G}$ showwound healing assays. The results indicate that overexpression of NRP1 promoted proliferation, migration, and invasion of 9810 cells.

\section{p-FAK/p-PI3-K/p-AKT Pathway Could Be One of the Possible Mechanisms Behind the Effects of NRPI on Cell Function}

To ascertain the signaling pathways that contribute to NRP1-related ICC transformation, we used WB to analyze the expression of key proteins including NK- $\kappa \mathrm{B}, \mathrm{p}-\mathrm{FAK}$, p-PI3-K, Paxillin, and p-AKT in common pathways, and the relative proteins were normalized to $\beta$-actin levels. As shown in Figure 6, downregulation of NRP1 in RBE cells by shRNA significantly reduced p-FAK, p-PI3-K, and p-AKT pathway activities compared to those in RBE-NC cells. Conversely, FAK, PI3-K, and AKT were clearly activated in $9810-\mathrm{OE}$ cells relative to $9810-\mathrm{NC}$ cells (Figure 6). The above results indicate that NRP1 depletion inhibits the activation of the p-FAK/p-PI3-K/p-AKT pathway, while high expression of NRP1 promotes the activation of the $\mathrm{p}-\mathrm{FAK} / \mathrm{p}-\mathrm{PI} 3-\mathrm{K} / \mathrm{p}-\mathrm{AKT}$ pathway.

\section{Discussion}

ICC is a highly invasive malignant tumor associated with an extremely poor prognosis, and radical resection is the only effective treatment presently. However, a vast majority of patients have different sites of invasion and metastasis at the time of identification. Therefore, early diagnosis and prevention of ICC invasion and metastasis are key steps to enhance the efficacy of treatment. ${ }^{26,28}$ Several reports have shown that mutations or elevated expression of NRP1 are correlated with many human malignancies. ${ }^{17,18,20,22,29}$ However, the expression of NRP1 and its possible role in the occurrence, development, and prognosis of ICC have been rarely discussed. The relationship between NRP1 expression and clinicopathology in ICC remains unclear.

In our research, based on a large number of organization samples from 291 ICC patients, we preliminarily studied the expression of NRP1 in ICC tissues and its 
A

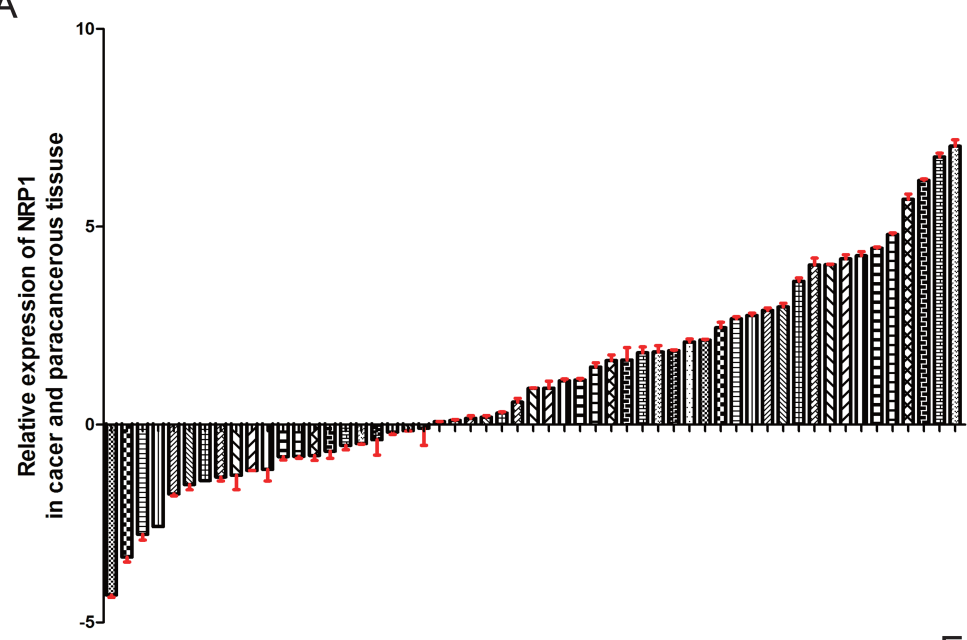

B

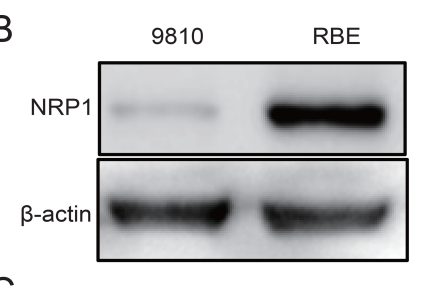

C

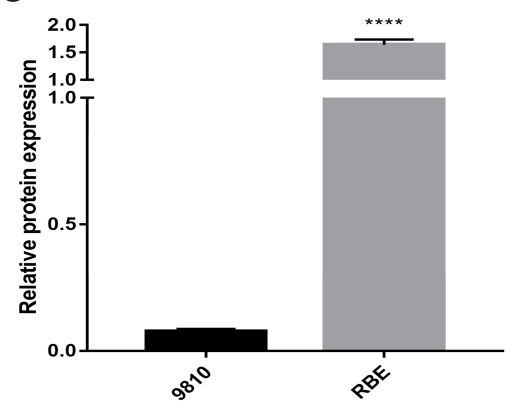

D
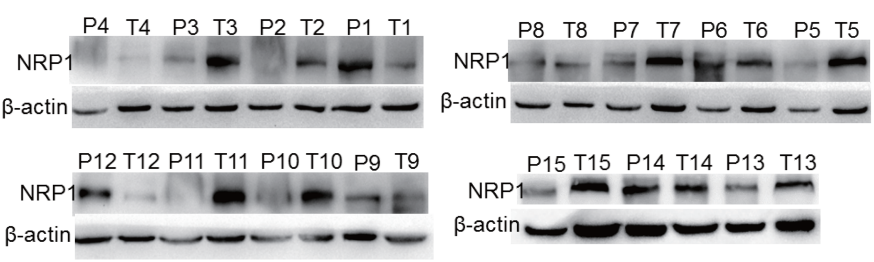

E

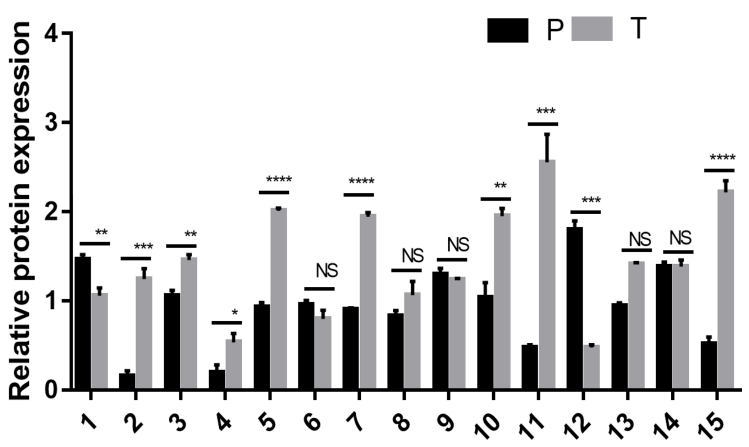

Figure 3 Expression difference of NRPI mRNAs and its proteins between ICC tissues and adjacent non-tumor tissues. (A) The expression level of NRPI mRNAs in 55 paired groups was detected using qRT-PCR (T, tumor tissue; P, adjacent non-tumor tissue). (B and $\mathbf{C}$ ) The expression level of NRPI proteins in ICC cell lines. (D and $\mathbf{E}$ ) Relative NRPI expression in 15 paired groups was analyzed using WB. $* P<0.05$, $* * P<0.01$, $* * * P<0.001$, and $* * * * P<0.000$ I.

correlation with prognosis and clinicopathological characteristics, and we found that NRP1 is associated with poor prognosis and may be a cancer promoting factor. Although a previous study ${ }^{30}$ reported that NRP1 is increased in human cholangiocarcinoma tissue, they chose NRP1 based on the previous gastric cancer study, and only pay attention to the effect of NRP1 silencing on cell proliferation. More perfectly, we focused on the effect of NRP1 silencing and overexpressing on proliferation, invasion, and migration of ICC cell lines. Moreover, this paper only measured the expression of NRP1 in five patients with cholangiocarcinoma, while our research measured the expression of NRP1 in 55 patients with carcinoma and paired normal tissues.

The important role of NRP1 in many malignant tumors has prompted us to investigate the clinical relevance, prognostic evaluation, and biological effects of NRP1 in human ICC. In our study, we demonstrated the relationship between high NRP1 expression and decreased overall survival and high risk of cumulative recurrence in ICC patients. Interestingly, although $35.40 \%$ of ICC patients had lower NRP1 levels in tumors than those in the adjacent non-tumor tissues, ICC generally showed an upregulation of NRP1, and high NRP1 expression indicated poor prognosis in all ICC patients. This suggested that NRP1 upregulation might occur in parallel contributing to the oncogenic process. It is worth mentioning that the NRP1 expression was significantly correlated with tumor number, and was an independent predictor of overall survival and cumulative recurrence. Patients with low NRP1 expression were 0.461 and 0.675 times less likely to suffer from tumor recurrence and death, respectively, than those with high NRP1 expression.

However, the specific effects of NRP1 on cell function are still unclear. Accordingly, further verification was essential. Based on the above results showing that the highest NRP1 expression was found in the RBE cell line and the lowest in 9810 cells, we overexpressed the NRP1 gene in the 9810 cell line and silenced it in the RBE cell line. The cell function experiments showed that 
A

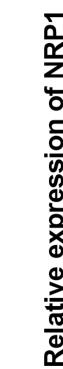

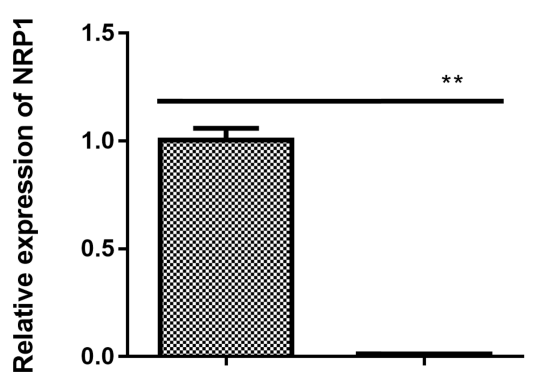

D

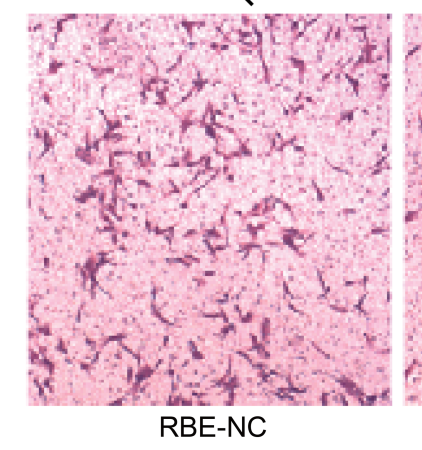

$\mathrm{F}$

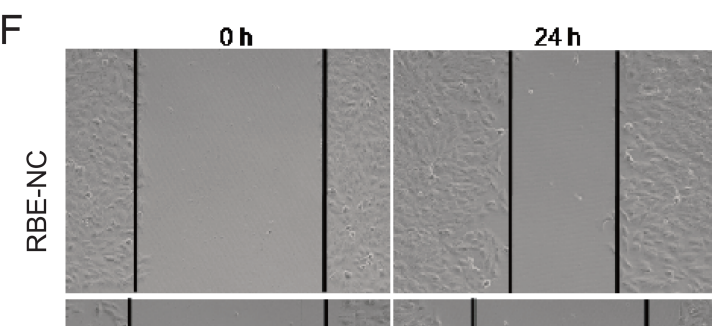

B

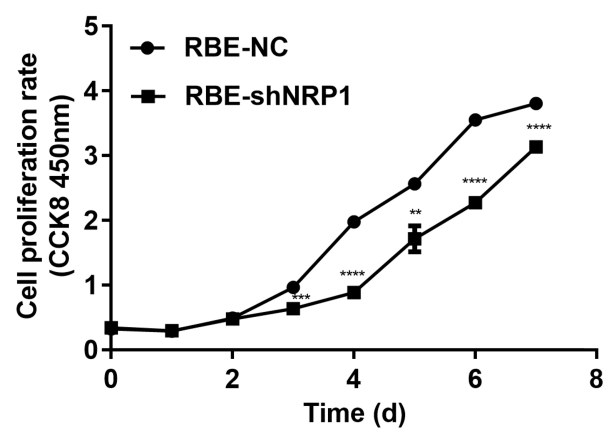

C

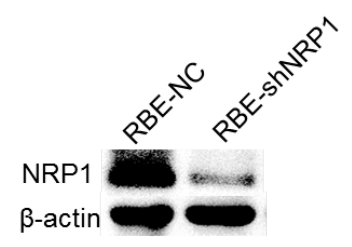

E

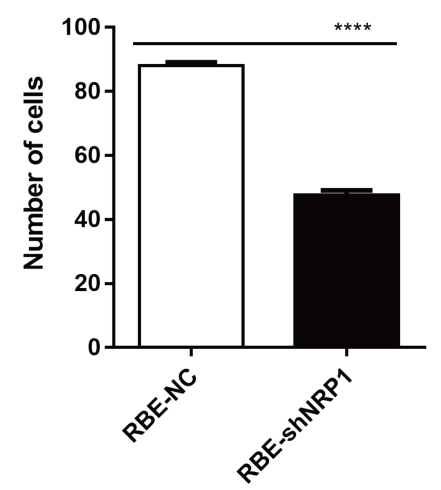

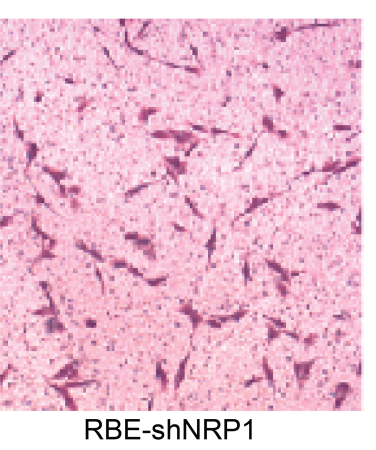
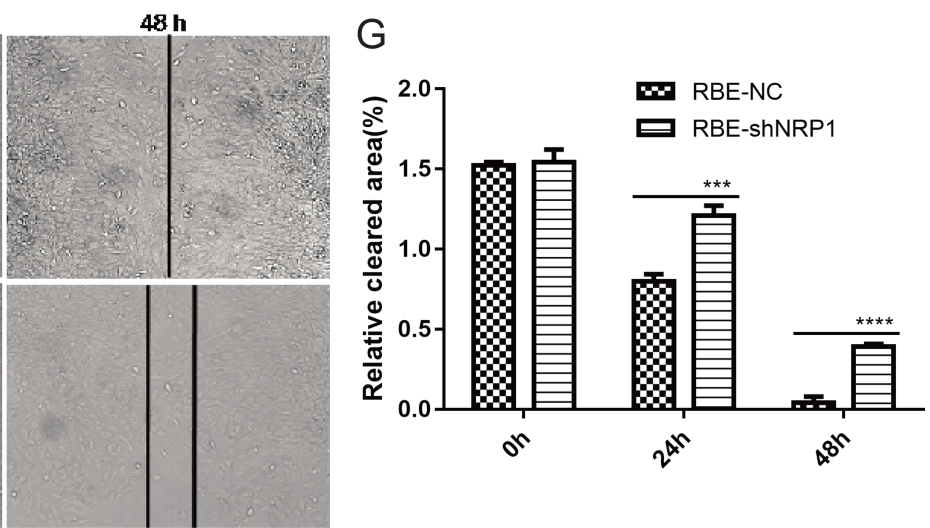

Figure 4 Cellular proliferation and migration comparison after NRPI knockdown in the RBE cell line. (A) NRPI expression levels in stably transfected RBE cells was confirmed by qRT-PCR. (B) CCK-8 assay confirmed that the proliferation ability of RBE-NC cells was better than that of RBE-shNRPI cells. (C) Western blotting confirmed NRPI expression levels in stably transfected ICC cells. (D and E) Transwell and (F and $\mathbf{G})$ Wound healing assays of RBE-shNRPI cells showed a considerable inhibitory effect on cell migration. Magnification, $\times 100$. $* * P<0.01$, *** $P<0.001$, and $* * * * * 0.0001$.

overexpression of NRP1 increased the proliferation, invasion, and migration abilities of the 9180 cell line. Conversely, knockdown of NRP1 in the RBE cell line impaired the proliferation and migration abilities of cells. These findings are in agreement with the findings of Zhu et $\mathrm{al}^{30}$ who showed that NRP1 depletion inhibits cell migration, proliferation, and angiogenesis.

Previous research has shown that focal adhesion kinase (FAK) is a cytoplasmic non-receptor tyrosine kinase and plays a pivotal role in cell migration, adhesion, and infiltration. ${ }^{31}$ FAK is involved in many cancer cells signaling pathways, which can transmit extracellular signals to cells via integrins and growth factor receptors. It is also associated with the PI3-K/AKT signaling pathway and can promote cytoskeletal reconstruction, formation, and renewal of adhesion plaques, and the expression of matrix metalloproteinase on the cell surface, thus promoting the migration and infiltration of cancer cells. ${ }^{32}$ Numerous research has shown that the FAK/PI3-K/AKT signaling pathway is intimately connected to the occurrence and development of ICC as well as the effectiveness of chemotherapy on ICC. ${ }^{33,34}$ In this study, we further 


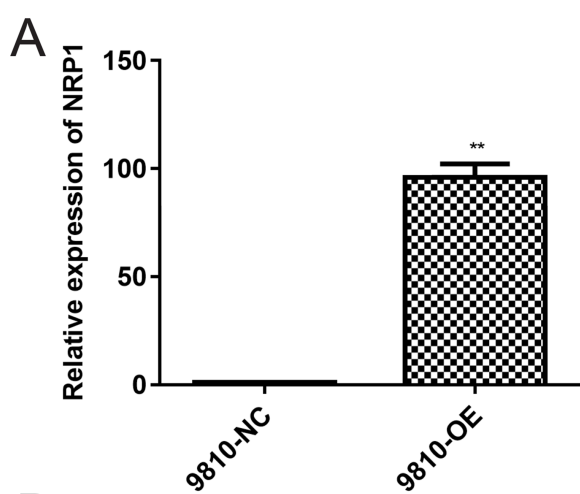

B

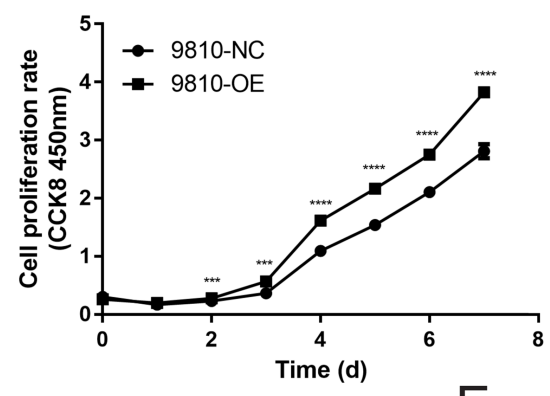

C

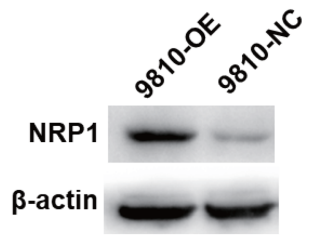

D

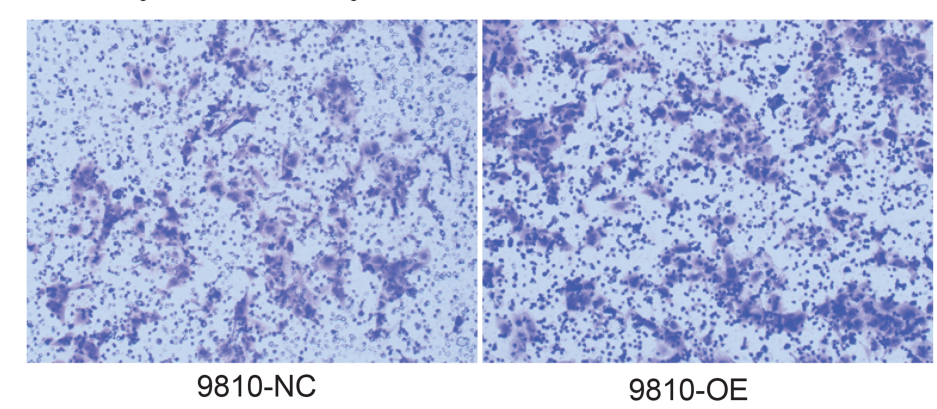

E
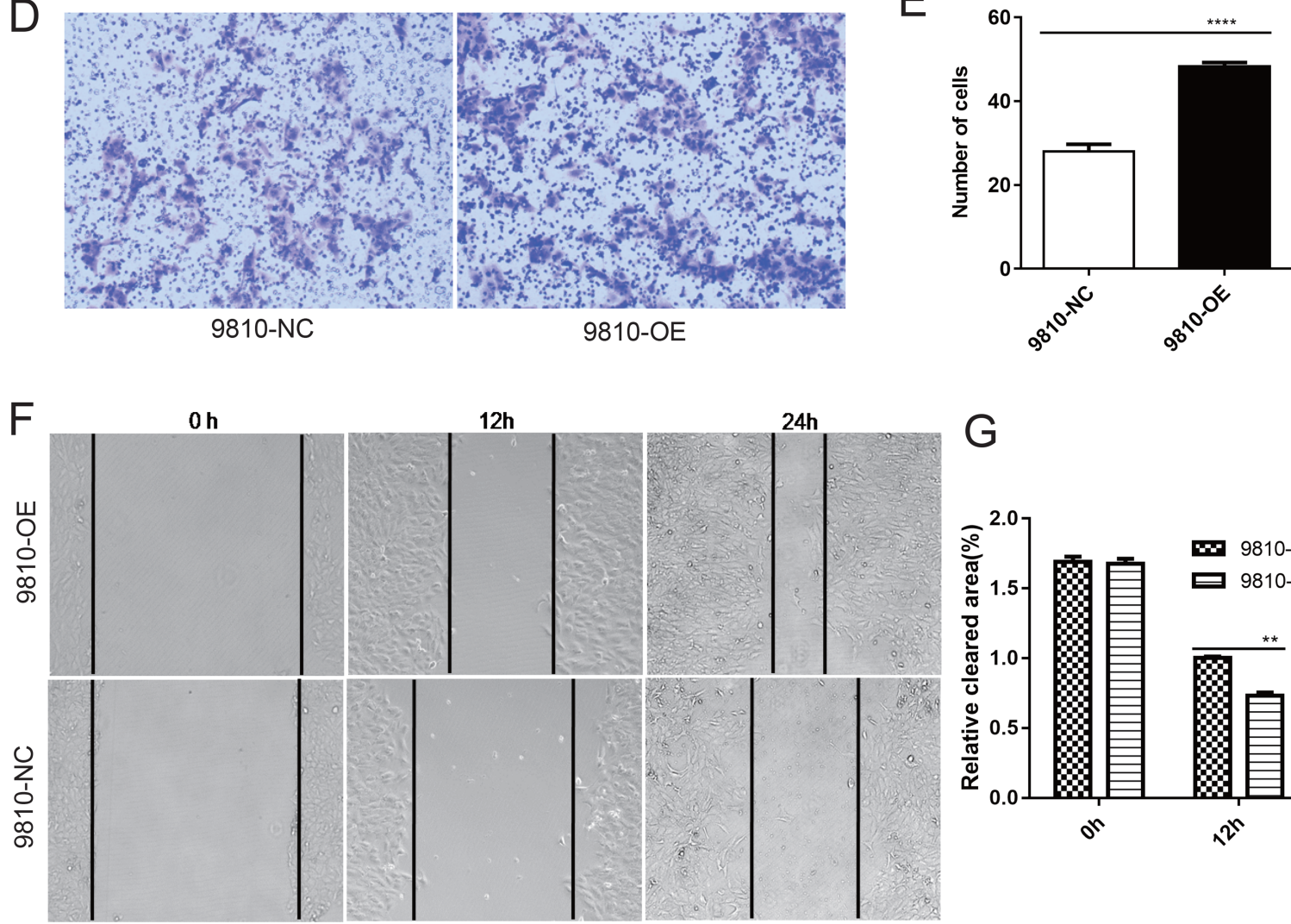

G

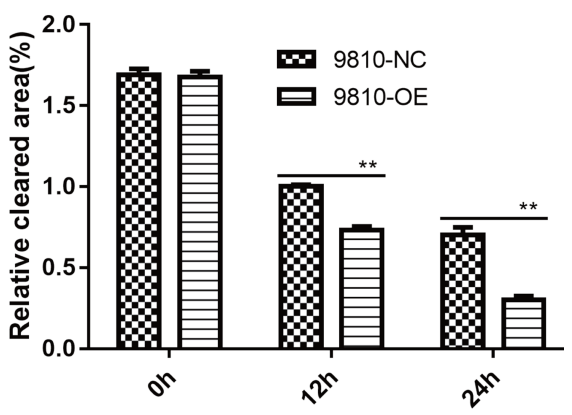

Figure 5 Cellular proliferation and migration comparison after NRPI overexpression in the 9180 cell line. (A) qRT-PCR confirmed NRPI expression levels in stably transfected ICC cells. (B) CCK-8 experiment confirmed that the proliferation ability of 98I0-OE-NRPI cells was better than that of 98I0-NC cells. (C) Western blotting confirmed NRPI expression levels in stably transfected ICC cells. (D and E) Transwell and (F and $\mathbf{G})$ wound healing assays of 98 I0-OE-NRPI cells showed a considerable inhibitory effect on cell migration capability. Magnification, $\times 100$. $* * P<0.01, * * * P<0.001$, and $* * * * P<0.0001$.

elucidated the signal transduction pathway and molecular mechanism of NRP1 in ICC. Based on the above findings, we speculated and verified that NRP1 may promote the proliferation, and migration of ICC cells via the $\mathrm{p}-\mathrm{FAK} / \mathrm{p}$ $\mathrm{PI} 3-\mathrm{K} / \mathrm{p}-\mathrm{AKT}$ pathway, which provides a new candidate target for inhibiting ICC proliferation and migration. Similarly, tolerance of ICC to conventional chemotherapy drugs is a major challenge during ICC treatment, and the mechanism of the tolerance may be related to the activation of the PI3-K/AKT signaling pathway. ${ }^{35}$ Reducing the expression of NRP1 and inhibiting PI3-K/AKT signaling may solve the problem of ICC tolerance to conventional chemotherapeutic drugs.

It is worth mentioning that previous studies have shown that NRP-1 can result in increased phosphorylation of FAK through the VEGF/VEGFR2 pathway, which is essential cancer metastasis. ${ }^{36,37}$ NRP-1 can also activate the $\mathrm{HGF} / \mathrm{c}-\mathrm{Met}$ pathway, leading to AKT activation and downregulation of $\mathrm{p} 27 .^{37,39}$ In addition to the PI3-K/AKT pathway, NRP-1 can promote cholangiocarcinoma cells proliferation and metastasis through the MEK/ERK pathway. ${ }^{30}$ Although the above signaling pathways were 


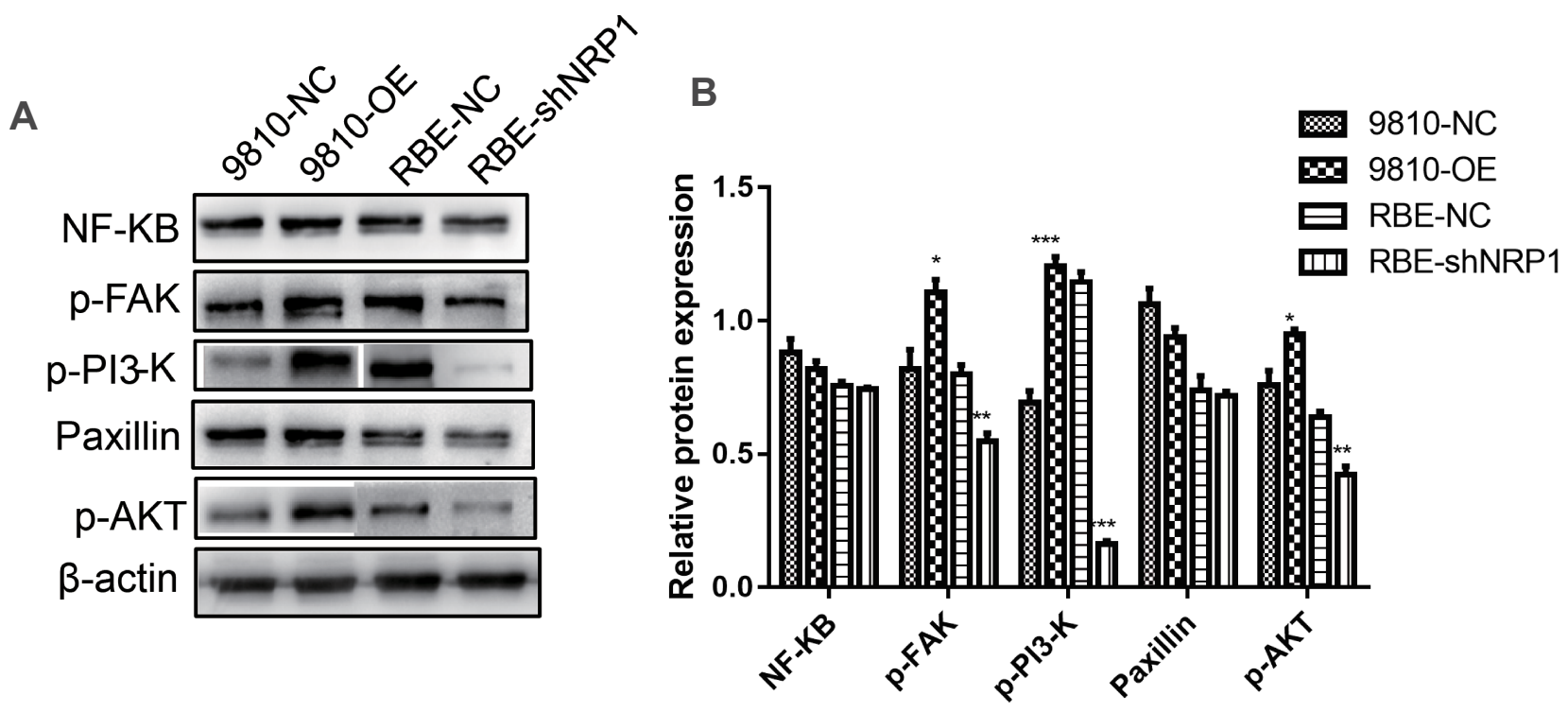

Figure 6 The expression level of NK-KB, p-FAK, p-PI3-K, Paxillin, and p-AKT. (A) The proteins in four types of ICC cells were detected using WB. (B) The relative proteins and the density of each band were normalized to $\beta$-actin levels. $* P<0.05, * * P<0.01$, and $* * * P<0.001$.

not investigated in this study, the crosstalk among the signaling pathways may promote the proliferation, invasion, and metastasis of ICC cells.

In conclusion, our study demonstrated that NRP1 is a valuable tumor-promoting and prognostic factor. The enigma behind NRP1 upregulation needs further studies to deeply investigate, which could be useful in the development of more effective targeted strategies to prevent ICC progression. To understand the function of NRP1 in ICC more comprehensively, more research will be needed, such as in vivo animal experiments and upstream and downstream signaling pathway experiments.

\section{Data Sharing Statement}

All data generated or analyzed during the present study are included in this published article.

\section{Ethics Approval and Consent}

All experiments conducted in the present study were approved by the Ethics Committee of the First Hospital of Lanzhou University (Gansu, China), and written informed consent was obtained.

\section{Acknowledgments}

This study was supported by grants from Gansu Province Science and Technology Major Project (1602FKDA001), Lanzhou Talent Innovation and Entrepreneurship Project (2016-RC-57), Gansu Health Industry Scientific Research
Plan Management Project (GWGL2014-57, GSWSKY2015-49), and Chengguan District Science and Technology Bureau Project (2017SHFZ0014). We also thank Dr. Wen-Bo Meng and Dr. Ping Yue for their professional contributions to this study.

\section{Author Contributions}

All authors made substantial contributions to conception and design, acquisition of data, or analysis and interpretation of data; took part in drafting the article or revising it critically for important intellectual content; gave final approval of the version to be published; and agree to be accountable for all aspects of the work.

\section{Disclosure}

The authors declare that they have no competing interests.

\section{References}

1. Khan AS, Dageforde LA. Cholangiocarcinoma. Surg Clin North Am. 2019;99(2):315-335. doi:10.1016/j.suc.2018.12.004

2. Lunsford KE, Javle M, Gaber AO, Vauthey JN, Ghobrial RM. Liver transplantation for locally advanced intrahepatic cholangiocarcinoma Reply. Lancet Gastroenterol. 2018;3(8):529-530. doi:10.1016/S24681253(18)30167-5

3. Fujita T, Razumilava N, Gores GJ. Liver transplantation for intrahepatic cholangiocarcinoma Reply. Lancet. 2014;384(9949):1182-1183. doi:10.1016/S0140-6736(14)61716-5

4. Buettner S, van Vugt JLA, Ijzermans JN, Groot Koerkamp B. Intrahepatic cholangiocarcinoma: current perspectives. Onco Targets Ther. 2017;10:1131-1142. doi:10.2147/OTT.S93629 
5. Wang K, Zhang H, Xia Y, Liu J, Shen F. Surgical options for intrahepatic cholangiocarcinoma. Hepatobiliary Surg Nutr. 2017;6 (2):79-90. doi:10.21037/hbsn.2017.01.06

6. Bray F, Ferlay J, Soerjomataram I, Siegel RL, Torre LA, Jemal A. Global cancer statistics 2018: GLOBOCAN estimates of incidence and mortality worldwide for 36 cancers in 185 countries. CA Cancer J Clin. 2018;68(6):394-424. doi:10.3322/caac.21492

7. Soejima Y, Takeuchi M, Akashi T, Sawabe M, Fukusato T. beta4 and beta6 integrin expression is associated with the subclassification and clinicopathological features of intrahepatic cholangiocarcinoma. Int J Mol Sci. 2018;19(4):1004. doi:10.3390/ijms 19041004

8. Yang SZ, Wang AQ, Du J, et al. Low expression of ARID1A correlates with poor prognosis in intrahepatic cholangiocarcinoma. World $J$ Gastroenterol. 2016;22(25):5814-5821. doi:10.3748/wjg.v22.i25.5814

9. Tsai Y-CI, Fotinou C, Rana R, et al. Structural studies of neuropilin-2 reveal a zinc ion binding site remote from the vascular endothelial growth factor binding pocket. FEBS J. 2016;283(10):1921-1934. doi: $10.1111 /$ febs. 13711

10. Wild JR, Staton CA, Chapple K, Corfe BM. Neuropilins: expression and roles in the epithelium. Int J Exp Pathol. 2012;93(2):81-103. doi:10.1111/j.1365-2613.2012.00810.x

11. Roy S, Bag AK, Singh RK, Talmadge JE, Batra SK, Datta K. Multifaceted role of neuropilins in the immune system: potential targets for immunotherapy. Front Immunol. 2017;8:1228. doi:10.3389/fimmu.2017.01228

12. Napolitano V, Tamagnone L. Neuropilins controlling cancer therapy responsiveness. Int $J$ Mol Sci. 2019;20(8):2049. doi:10.3390/ ijms20082049

13. Hu C, Jiang X. Role of NRP-1 in VEGF-VEGFR2-independent tumorigenesis. Target Oncol. 2016;11(4):501-505. doi:10.1007/ s11523-016-0422-0

14. Prud'homme GJ, Glinka Y. Neuropilins are multifunctional coreceptors involved in tumor initiation, growth, metastasis and immunity. Oncotarget. 2012;3(9):921-939. doi:10.18632/oncotarget.626

15. Torres-Salido MT, Sanchis M, Sole C, et al. Urinary neuropilin-1: a predictive biomarker for renal outcome in lupus nephritis. Int $\mathrm{J} \mathrm{Mol}$ Sci. 2019;20(18):4601. doi:10.3390/ijms20184601

16. Chaudhary B, Khaled YS, Ammori BJ, Elkord E. Neuropilin 1: function and therapeutic potential in cancer. Cancer Immunol Immunother. 2014;63(2):1-99. doi:10.1007/s00262-013-1500-0

17. Bergé M, Allanic D, Bonnin P, et al. Neuropilin-1 is upregulated in hepatocellular carcinoma and contributes to tumour growth and vascular remodelling. J Hepatol. 2011;55(4):866-875. doi:10.1016/j. jhep.2011.01.033

18. Peng Y, Liu Y-M, Li L-C, Wang -L-L, Wu X-L. MicroRNA-338 inhibits growth, invasion and metastasis of gastric cancer by targeting NRP1 expression. PLoS One. 2014;9(4):e94422-e94422. doi:10.1371/journal.pone.0094422

19. Glinka Y, Mohammed N, Subramaniam V, Jothy S, Prud'homme GJ. Neuropilin-1 is expressed by breast cancer stem-like cells and is linked to NF-kappaB activation and tumor sphere formation. Biochem Biophys Res Commun. 2012;425(4):775-780. doi:10.1016/ j.bbrc.2012.07.151

20. Naik A, Al-Zeheimi N, Bakheit CS, et al. Neuropilin-1 associated molecules in the blood distinguish poor prognosis breast cancer: a cross-sectional study. Sci Rep. 2017;7(1):3301. doi:10.1038/s41598017-03280-0

21. Tse BWC, Volpert M, Ratther E, et al. Neuropilin-1 is upregulated in the adaptive response of prostate tumors to androgen-targeted therapies and is prognostic of metastatic progression and patient mortality. Oncogene. 2017;36(24):417-3427. doi:10.1038/onc.2016.482

22. Shi S, Lu Y, Qin Y, et al. miR-1247 is Correlated with Prognosis of Pancreatic Cancer and Inhibits Cell Proliferation by Targeting Neuropilins. Curr Mol Med. 2014;14(3):316-327. doi:10.2174/ 1566524014666140228120014
23. Bi C, Liu M, Rong W, et al. High Beclin-1 and ARID1A expression corelates with poor survival and high recurrence in intrahepatic cholangiocarcinoma: a histopathological retrospective study. $B M C$ Cancer. 2019;19(1):213. doi:10.1186/s12885-019-5429-3

24. Gao ZF, Wu YN, Bai ZT, Zhang L, Zhou Q, Li X. Tumor-suppressive role of HACE1 in hepatocellular carcinoma and its clinical significance. Oncol Rep. 2016;36(6):3427-3435. doi:10.3892/or.2016.5205

25. Ohara K, Horibe T, Kohno M, Kawakami K. Characterization of antilytic peptide antibody: application for the detection of lyticbased hybrid peptide in serum samples. J Pept Sci. 2011;17(7):493498. doi:10.1002/psc.1349

26. Rahnemai-Azar AA, Weisbrod AB, Dillhoff M, Schmidt C, Pawlik TM. Intrahepatic cholangiocarcinoma: current management and emerging therapies. Expert Rev Gastroenterol Hepatol. 2017;11 (5):439-449. doi:10.1080/17474124.2017.1309290

27. Zhu AX, Hezel AF. Development of molecularly targeted therapies in biliary tract cancers: reassessing the challenges and opportunities. Hepatology. 2011;53(2):695-704. doi:10.1002/hep.24145

28. Guion-Dusserre JF, Lorgis V, Vincent J, Bengrine L, Ghiringhelli F. FOLFIRI plus bevacizumab as a second-line therapy for metastatic intrahepatic cholangiocarcinoma. World J Gastroenterol. 2015;21 (7):2096-2101. doi:10.3748/wjg.v21.i7.2096

29. Ueyama H, Horibe T, Nakajima O, Ohara K, Kohno M, Kawakami K. Semaphorin 3A lytic hybrid peptide binding to neuropilin-1 as a novel anti-cancer agent in pancreatic cancer. Biochem Biophys Res Commun. 2011;414(1):60-66. doi:10.1016/j.bbrc.2011.09.021

30. Zhu H, Jiang X, Zhou X, et al. Neuropilin-1 regulated by miR-320 contributes to the growth and metastasis of cholangiocarcinoma cells. Liver Int. 2018;38(1):125-135. doi:10.1111/liv.13495

31. Zhou J, Yi Q, Tang L. The roles of nuclear focal adhesion kinase (FAK) on cancer: a focused review. J Exp Clin Cancer Res. 2019;38 (1):250. doi:10.1186/s13046-019-1265-1

32. Lee BY, Timpson P, Horvath LG, Daly RJ. FAK signaling in human cancer as a target for therapeutics. Pharmacol Ther. 2015;146:132149. doi:10.1016/j.pharmthera.2014.10.001

33. Chen M-H, Chiang K-C, Cheng C-T, et al. Antitumor activity of the combination of an HSP90 inhibitor and a PI3K/mTOR dual inhibitor against cholangiocarcinoma. Oncotarget. 2014;5(9):2372-2389. doi:10.18632/oncotarget.1706

34. Yothaisong S, Dokduang H, Techasen A, et al. Increased activation of $\mathrm{PI} 3 \mathrm{~K} / \mathrm{AKT}$ signaling pathway is associated with cholangiocarcinoma metastasis and PI3K/mTOR inhibition presents a possible therapeutic strategy. Tumor Biol. 2013;34(6):3637-3648. doi:10.1007/s13277013-0945-2

35. McRee AJ, Sanoff HK, Carlson C, Ivanova A, O’Neil BH. A Phase I trial of mFOLFOX6 combined with the oral PI3K inhibitor BKM120 in patients with advanced refractory solid tumors. Invest New Drugs. 2015;33(6):1225-1231. doi:10.1007/s10637-015-0298-3

36. Hamerlik P, Lathia JD, Rasmussen R, et al. Autocrine VEGF VEGFR2 - neuropilin-1 signaling promotes glioma stem-like cell viability and tumor growth. J Exp Med. 2012;209(3):507-520. doi:10.1084/jem.20111424

37. Barr MP, Gray SG, Gately K, et al. Vascular endothelial growth factor is an autocrine growth factor, signaling through neuropilin-1 in non-small cell lung cancer. Mol Cancer. 2015;14:45. doi:10.1186/ s12943-015-0310-8

38. Shang N, Arteaga M, Zaidi A, et al. FAK is required for c-Met/betacatenin-driven hepatocarcinogenesis. Hepatology. 2015;61(1):214226. doi:10.1002/hep. 27402

39. Barat S, Bozko P, Chen X, et al. Targeting c-MET by LY2801653 for treatment of cholangiocarcinoma. Mol Carcinog. 2016;55(12):20372050. doi:10.1002/mc.22449 


\section{Publish your work in this journal}

Cancer Management and Research is an international, peer-reviewed open access journal focusing on cancer research and the optimal use of preventative and integrated treatment interventions to achieve improved outcomes, enhanced survival and quality of life for the cancer patient.
The manuscript management system is completely online and includes a very quick and fair peer-review system, which is all easy to use. Visit http://www.dovepress.com/testimonials.php to read real quotes from published authors. 